\title{
Development of New Lesions of Hidradenitis Suppurativa on a Caesarean Section Scar: A Manifestation of the Koebner Phenomenon?
}

\author{
Efthimia Agiasofitou ${ }^{a} \quad$ Theodora Kanni $^{a} \quad$ Eftychia Platsidaki ${ }^{a}$ \\ Vasiliki Tzanetakou $^{\mathrm{a}}$ Stamatios Gregoriou ${ }^{\mathrm{b}}$ Dimitrios Rigopoulos $^{\mathrm{b}}$ \\ George Kontochristopoulos ${ }^{a}$ \\ ${ }^{a}$ Department of Dermatology and Venereology, Andreas Sygros Hospital, Athens, Greece; ${ }^{\mathrm{b}} 1$ st Department of \\ Dermatology and Venereology, University of Athens Medical School, Athens, Greece
}

\section{Established Facts}

- The Koebner phenomenon has been described following a surgical procedure in various dermatoses, including psoriasis, vitiligo, and lichen planus.

- Koebnerization in hidradenitis suppurativa (HS) has recently been observed in patients in whom new lesions developed as a result of trauma.

\section{Novel Insights}

- The Koebner phenomenon following the scarring process on a cesarean section site has not previously been reported.

- Avoidance of external trauma could be included in general measures of HS treatment.

- Disease recurrence should be considered for patients treated with surgical excision of HS lesions.

\section{Keywords}

Hidradenitis suppurativa · Cesarean section · Koebner phenomenon

\section{Abstract \\ Hidradenitis suppurativa (HS) is a chronic, inflammatory, re- current skin disorder of the hair follicle. Trauma, mechanical pressure, or friction could have an important role in the pathogenesis of HS. We present a patient with HS who de-}

veloped new HS lesions shortly after a cesarean section, which could be explained by the Koebner phenomenon. Koebnerization in HS has recently been observed in patients in whom new lesions developed at an ectopic location free from apocrine glands as a result of trauma. More studies need to be conducted to shed light on the possible relation between HS and surgical trauma. Avoidance of external trauma could be included in general measures of HS treatment.

(c) 2020 S. Karger AG, Basel karger@karger.com www.karger.com/sad

(c) 2020 S. Karger AG, Basel

Karger"
Eftychia Platsidaki, MD

Department of Dermatology and Venereology Andreas Sygros Hospital

5 Ionos Dragoumi Str, GR-161 21 Athens (Greece)

platsidakieft@yahoo.com 


\section{Introduction}

Hidradenitis suppurativa (HS) is a chronic, inflammatory, recurrent skin disorder of the hair follicle. HS usually begins after puberty and is characterized by inflamed, deeply seated lesions located in anatomical areas rich in apocrine glands, predominantly around the axillae, groins, and the anogenital area. HS seems to affect the global population. Prevalence in the US and Europe ranges from 0.97 to $4 \%$. The pathogenesis of HS is not fully understood. It appears to be complex and multifactorial. The main factor is the obstruction of the hair follicle caused by hyperkeratinization of the follicular infundibulum. Genetic predisposition, hormonal factors, microorganisms, obesity, smoking, and irritant factors (trauma and mechanical pressure) are also implicated $[1,2]$. In this case report, we describe a patient with HS who developed new HS lesions shortly after a cesarean section.

\section{Case Report}

A 34-year-old female patient suffering from HS (stage Hurley II) since she was 18 years of age was admitted due to the appearance of new nodules and fistulae with intermittent purulence on a cesarean section incision site that had been performed 5 months earlier (Fig. 1). The lesions appeared 2-3 weeks after her second cesarean section. The patient reported similar lesions following her first cesarean section, while during pregnancy, remission of $\mathrm{HS}$ had been observed. On physical examination, both axillary areas were also found to be affected (Fig. 2a, b). The patient displayed hypertrophic scars on the right axilla, whereas an inflammatory nodule, draining fistula, and multiple pustules were present on the left axilla. HS medical history showed repeated courses of oral antibiotics to treat disease exacerbations. A family history of HS was reported. More specifically, her father was also affected by HS. The patient was a smoker with a BMI of 31.2. According to her personal history, the patient had undergone cholocystectomy and surgery for esophageal achalasia. For this current episode of HS, doxycycline at a dose of $100 \mathrm{mg}$ twice daily and application of topical antiseptic (octenidine) and clindamycin/benzoyl peroxide gel were recommended.

\section{Discussion}

Trauma, mechanical pressure, or friction could have an important role in the pathogenesis of HS. The development of HS lesions following the scarring process on a cesarean section site has not previously been reported and could be explained by the Koebner phenomenon. The Koebner phenomenon by definition indicates the appearance of a new lesion at the site of skin injury in patients

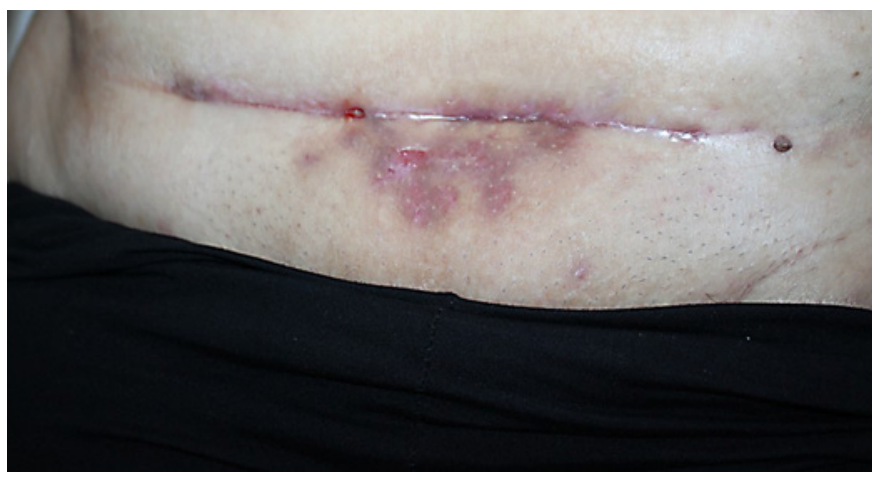

Fig. 1. Nodules and fistulae with intermittent purulence on the cesarean section incision site.
Fig. 2. a, b Inflammatory nodules, draining fistulae, and hypertrophic scars on the right and left axillae.

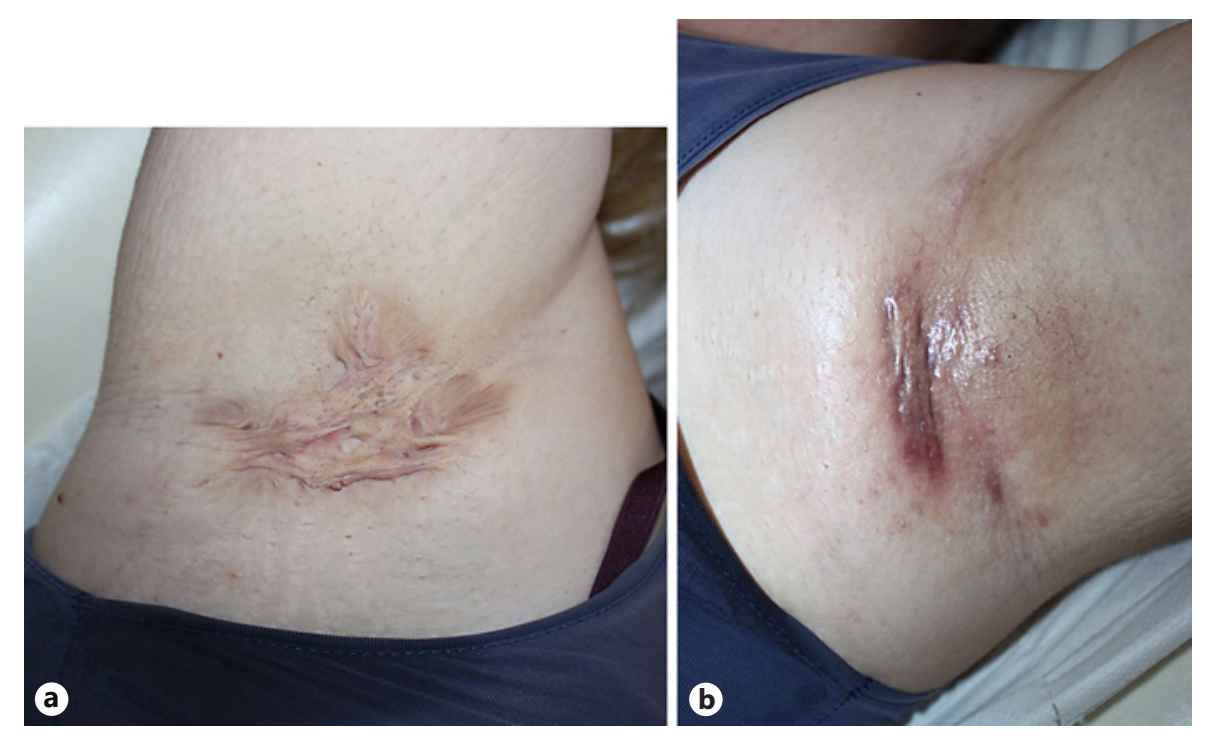


with a history of skin disease. It has been described following a surgical procedure in various dermatoses, including psoriasis, vitiligo, and lichen planus. Koebnerization in HS has recently been observed in patients in whom new lesions developed at an ectopic location free from apocrine glands as a result of trauma [3-5]. Epidermal injury following surgery strongly increases the release of proinflammatory cytokines resulting in the plugging of the follicle opening, follicle dilation, and the whole cascade of the development of new HS lesions $[6,7]$. The concept of an immunocompromised district, as already described for other diseases, could be also considered [8]. A skin area more vulnerable than the rest of the body for genetic or acquired reasons may present as an area prone to develop infections, tumors, or dysimmune reactions. More studies need to be conducted to shed light on the possible relation between HS and surgical trauma. Avoidance of external trauma could be included in general measures of HS treatment. Additionally, the possibility of disease recurrence should be considered for patients treated with surgical excision of HS lesions.

\section{Statement of Ethics}

The study was approved by the ethics committee of the hospital. The patient's consent to publish photos and details of the case has been obtained.

\section{Disclosure Statement}

The authors declare that they have no conflicts of interest to disclose.

\section{Funding Sources}

The authors did not receive any funding.

\section{Author Contributions}

All authors contributed to the design of the work, acquisition, analysis, and interpretation of data, critically revised the manuscript for important intellectual content, and approved the final version.

\section{References}

1 Boer J, Mihajlovic D. Boils at frictional locations in a patient with hidradenitis suppurativa. Acta Dermatovenerol Croat. 2016 Dec; 24(4):303-4.

2 Maher M, Larsen L. A case of radiation-induced localized exacerbation of hidradenitis suppurativa. JAAD Case Rep. 2016 Jan;2(1): 44-6.

3 de Winter K, van der Zee HH, Prens EP. Is mechanical stress an important pathogenic factor in hidradenitis suppurativa? Exp Dermatol. 2012 Mar;21(3):176-7.
4 Boer J. Should hidradenitis suppurativa be included in dermatoses showing koebnerization? Is it friction or fiction? Dermatology. 2017;233(1):47-52.

5 Boer J. Resolution of hidradenitis suppurativa after weight loss by dietary measures, especially on frictional locations. J Eur Acad Dermatol Venereol. 2016 May;30(5):895-6.
6 Musumeci ML, Pavone A, Barresi S, Scilletta A, Micali G. Koebner phenomenon of hidradenitis suppurativa on a surgical scar. Int Wound J. 2019 Oct;16(5):1231-3.

7 Boer J, Nazary M, Riis PT. The Role of Mechanical Stress in Hidradenitis Suppurativa. Dermatol Clin. 2016 Jan;34(1):37-43.

8 Marasca C, Annunziata MC, Cantelli M, Marasca D, Fabbrocini G. Marjolin's ulcer in a paraplegic man: A further example of the immunocompromised district. Int Wound J. 2019 Apr;16(2):574-5. 